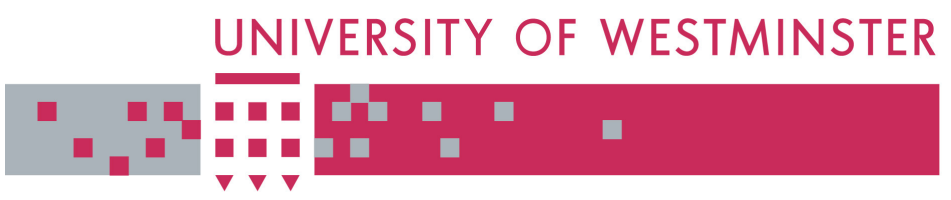

\title{
WestminsterResearch
}

http://www.wmin.ac.uk/westminsterresearch

\section{A tool for studying the effects of residents' attributes on patterns of length of stay in long-term care.}

\author{
Haifeng Xie \\ Thierry Chaussalet \\ Peter Millard
}

Cavendish School of Computer Science

Copyright (C [2005] IEEE. Reprinted from Proceedings of the 18th IEEE Symposium on Computer-Based Medical Systems: 23-25 June 2005, Dublin, Ireland. pp. 473-478.

This material is posted here with permission of the IEEE. Such permission of the IEEE does not in any way imply IEEE endorsement of any of the University of Westminster's products or services. Internal or personal use of this material is permitted. However, permission to reprint/republish this material for advertising or promotional purposes or for creating new collective works for resale or redistribution must be obtained from the IEEE by writing to pubs-permissions@ieee.org. By choosing to view this document, you agree to all provisions of the copyright laws protecting it.

The WestminsterResearch online digital archive at the University of Westminster aims to make the research output of the University available to a wider audience. Copyright and Moral Rights remain with the authors and/or copyright owners. Users are permitted to download and/or print one copy for non-commercial private study or research. Further distribution and any use of material from within this archive for profit-making enterprises or for commercial gain is strictly forbidden.

Whilst further distribution of specific materials from within this archive is forbidden, you may freely distribute the URL of WestminsterResearch.

(http://www.wmin.ac.uk/westminsterresearch).

In case of abuse or copyright appearing without permission e-mail wattsn@wmin.ac.uk. 


\title{
A Tool for Studying the Effects of Residents' Attributes on Patterns of Length of Stay in Long-term Care
}

\author{
Haifeng Xie, Thierry Chaussalet, Peter Millard \\ Health and Social Care Modelling Group \\ Cavendish School of Computer Science \\ University of Westminster, London, UK \\ $\{$ h.f.xie, chausst $\} @ w m i n . a c . u k$, phmillard@tiscali.co.uk
}

\begin{abstract}
Understanding the differential pattern of length of stay (LOS) in long-term care (LTC) due to residents' attributes has important practical implications in the management of long-term care. In this paper, we extend a previously developed modelling approach to incorporate residents' attributes. Two applications using data collected by a local authority in England are presented to demonstrate the potential use of this extension. In the study of possible difference in LOS pattern due to gender, our model provides quantitative support to the observations that male residents admitted to NC take more time to settle down and have poorer short-term survival prospect than female residents.
\end{abstract}

\section{Introduction}

Older people often experience a decline in physical and mental health, and activities of daily living, for example, feeding, toileting and self care, can become difficult. In cases where older persons can no longer be cared for at home, assistance is required from agencies providing longterm care (LTC).

Local authorities in Great Britain play an important role in the delivery of LTC. Under the 1990 NHS and Community Care Act and the Care Standard Act 2000, local authorities are responsible for the placement and finance of adults in publicly funded residential care (RC) and nursing care (NC) that conforms to national standards. The UK government is planning to fine local authorities for failing to provide vacancies in institutional care homes for hospital discharges [7]. Therefore, it is important for local authorities to have a sound understanding of the behaviour of the LTC system.

The focus is on publicly funded residents in institutional LTC (ILTC). Since most local authorities have means of determining suitable care placements for applicants requiring public funds, these admissions will reflect residents' physical conditions and needs.

In this paper, we extend a modelling approach previously reported [9] to take into account the attributes of individuals, for example, their age and gender, etc. We demonstrate the potential use of this extended model using two case studies. 


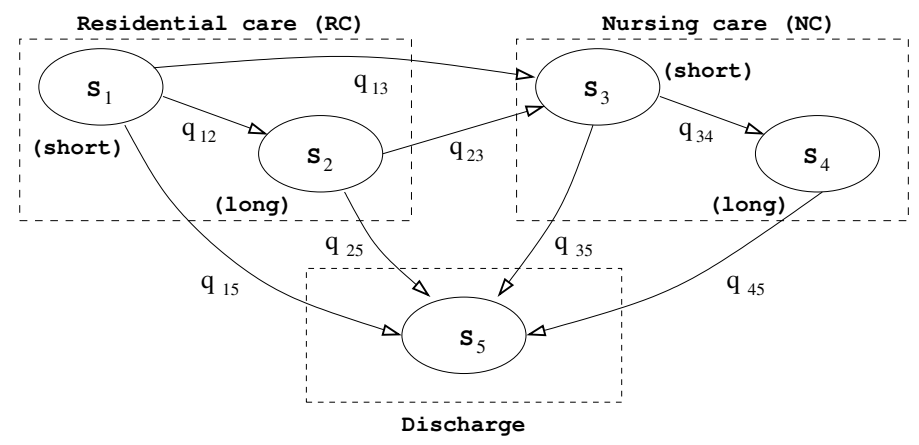

Figure 1. Markov model for the movement of publicly funded residents in ILTC.

\section{Method}

\subsection{Movement of elderly people in institutional long-term care}

Research in the UK shows that the mortality rate for publicly funded residents in NC is particularly high in the first few months and then gradually levels out [1,6]. This observation supports the notion of different phases in the stay of residents in care homes. In a previous study [9], we presented a continuous-time Markov model for the flow of elderly residents within and between RC and NC. Briefly, the model (see Figure 1) uses a combination of a short-stay state and a longstay state to capture the flow of residents through each type of care. For instance, a person admitted to RC might stay for a short period of time, then is either discharged (predominately by death) or transferred to NC; or the person might settle down and become a long-stay resident in RC. However, the state space of the Markov model is aggregated [4] due to the un-observability of the actual shortstay and long-stay states, i.e., we observe a person is in RC but do not know whether the person is in short-stay or long-stay state. A procedure for fitting such a model to observation data was developed (for details see $[8,9]$ ).

\subsection{Incorporating residents' attributes}

The Markov model described in the previous section assumes that transition rates (e.g., $q_{12}$ ) are constant for all residents in the system. This can be restrictive, considering the vast diversity that exists among individuals entering the ILTC system. It would be desirable for the model to take into account the attributes of individuals.

Assume there exist measurements on $p$ covariates (or attributes) $X_{1}, \ldots, X_{p}$ for each resident upon admission to each type of care. For the $k$ th individual, with observation $\boldsymbol{x}_{k}=$ $\left(1, x_{k 1}, x_{k 2}, \ldots, x_{k p}\right)$, the transition probability between states $i$ and $j(i \neq j)$, where transition from $i$ to $j$ is possible, can be written as

$$
q_{i j, k}=\exp \left(\boldsymbol{\beta}_{i j}^{\mathrm{T}} \boldsymbol{x}_{k}\right),
$$

where $\boldsymbol{\beta}_{i j}$ is a vector of coefficients. Therefore, an attribute that has significant effect on a transition rate (hence on LOS) will have its corresponding coefficient significantly different from zero. The model parameters $\boldsymbol{\beta}_{i j}$ are estimated by numerical maximisation of the likelihood function [9].

This approach, which consists in incorporating covariates into the Markov model via a loglinear function is relatively straightforward, and similar to that taken by [3].However, the number of parameters to be estimated grow rapidly as the number of covariates increases. 

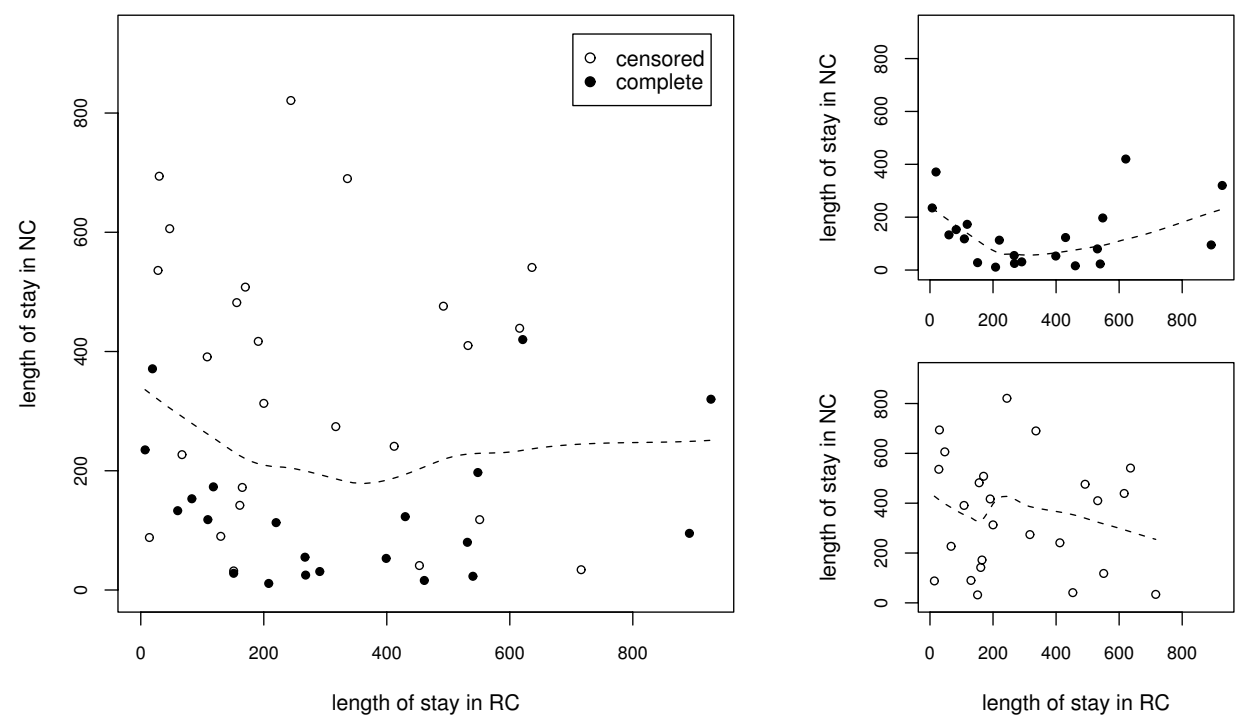

Figure 2. Scatter plot of LOS in RC and NC among NC residents who were transferred from RC in the Merton dataset. Grouping is based on whether a resident was alive, i.e., the observation is censored, or had been discharged, i.e., the observation is complete, on 1 April 2001. The dotted lines correspond to the locally fitted smoothing curves.

\section{Application}

We demonstrate the usefulness of the model that incorporates residents' attributes with two applications. The data used in the applications were provided by the Housing and Social Service Departent of the London Borough of Merton, UK. The dataset contains information on LOS and movement of publicly funded residents who were admitted to ILTC between April 1997 to April 2001. In all, there were 935 records concerning 889 residents (for detail description of the dataset, see [9]).

Given the dataset, it was found [9] that there were one state in RC and two states in NC in the Markov model of residents movements presented in section 2.1, i.e. $q_{12}=q_{23}=q_{25}=0$ in Figure 1.

\subsection{Difference in LOS pattern among residents in NC}

The Markov property implies that the LOS pattern for residents directly admitted to NC is the same as those transferred from RC. However, there might be reasons to believe that a person who spent a long time in RC might also stay for a long time in NC. We use our model to investigate possible differences in LOS pattern among NC residents, i.e., between those admitted to NC directly and those transferred from RC.

We treated LOS in RC (being zero for those who were admitted to NC directly) as an attribute of a resident. Two different ways of incorporating this attribute into the model were considered - namely, Model 1: LOS in RC as a continuous variable; Model 2: previous presence in RC as a binary variable, where " 1 " represents having stayed in RC prior to admission to NC. Therefore, 
Table 1. Estimated parameters from the Merton dataset.

\begin{tabular}{c|r|c|c}
\hline \hline parameter & estimate & standard error & 95\% confidence interval \\
\hline$a_{13}$ & -8.622 & 0.189 & $(-8.992,-8.251)$ \\
$a_{34}$ & -4.210 & 0.397 & $(-4.988,-3.433)$ \\
$a_{15}$ & -7.120 & 0.079 & $(-7.275,-6.966)$ \\
$a_{35}$ & -5.353 & 0.153 & $(-5.652,-5.054)$ \\
$a_{45}$ & -6.711 & 0.110 & $(-6.927,-6.495)$ \\
$b_{13}$ & 0.827 & 0.302 & $(0.235,1.419)$ \\
$b_{34}$ & -0.991 & 0.428 & $(-1.831,-0.152)$ \\
\hline \hline
\end{tabular}

together with the base model (i.e., not taking into account previous LOS in RC, denoted as Model 0), there are Three models under consideration.

Comparisons between these (nested) models, based on the likelihood ratio test (LRT) statistics, did not show that the incorporation of the attribute provided significant improvement over the base model, judging at 5\% significant level (Model 0 vs. Model 1: $X^{2}=5.36, p=0.1473$; Model 0 vs. Model 2: $\left.X^{2}=4.60, p=0.2035\right)$. Therefore, the fitted result did not suggest that there was a significant difference in LOS pattern between NC residents transferred from RC and those admitted directly. This finding was also consistent with the result of a formal hypothesis test (log-rank test, $W_{L}=1.875, p=0.1709$ ) and a visual inspection of the data (see Figure 2).

\subsection{Difference in LOS pattern due to gender}

A recent national survey in care homes in England showed that survival prospect was particular poor for publicly funded male residents admitted to NC, especially in the first few months following admission [1]. We can use our model to study whether there is a significant difference in LOS pattern between male and female residents in ILTC, and to quantify this difference if it exists. The London Borough of Merton also provided information on gender for the group of residents presented in our dataset. However, due to missing information on some of the residents, the number of records used in this application was slightly reduced to 861 .

We parameterised the model to incorporate gender as an attribute. More specifically, the transition rate between state $i$ and state $j$ (where transition from states $i$ to state $j$ is possible) for the $k$ th person is written as $q_{i j, k}=\exp \left(a_{i j}+b_{i j} x_{k}\right)$, where $x_{k}=1$ if the person is male and $x_{k}=0$ otherwise.

The model was fitted to the Merton dataset with the attribute entering each transition rate in a sequential manner. Competing models were tested by the LRT statistic. The final model showed that gender had a significant influence on transition rates $q_{13}$ and $q_{34}$ (see Table 1).

The average LOS for male residents in RC is estimated to be 819 days or 2.2 years (estimated by $\left.\left(e^{a_{13}+b_{13}}+e^{a_{15}}\right)^{-1}\right)$, which is shorter than that of female residents (1011 days or 2.8 years). Upon leaving RC, $18 \%$ female residents and $34 \%$ male residents (estimated by $e^{a_{13}+b_{13}} /\left(e^{a_{13}+b_{13}}+e^{a_{15}}\right)$ ) will be transferred to NC. In other words, on average, male residents have a shorter stay in RC, and are $16 \%$ more likely to make the transition from $\mathrm{RC}$ to $\mathrm{NC}$ than female residents.

The fitted result also suggests that there exist significant differences in short-stay patterns between genders. On average, male residents stay almost twice as long as female residents in the short-stay state ( 98 days for male, and 51 days for female). However, after this settle-in period, male residents in $\mathrm{NC}$ are $22 \%$ less likely to make the transition from short-stay to long-stay, i.e., male residents are more likely to be discharged earlier than female residents. 

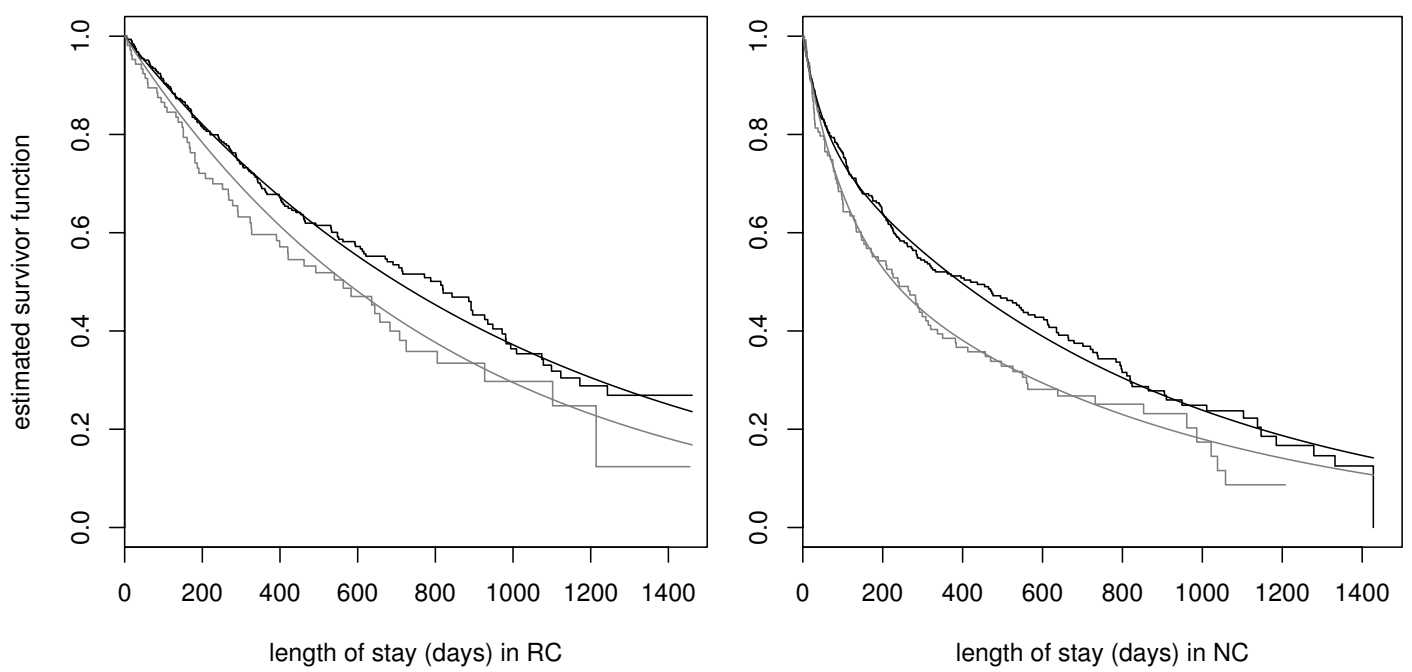

Figure 3. Kaplan-Meier estimated (jagged line) and Markov model fitted (smooth line) survivor curves of LOS for female (black colour) and male (grey colour) residents in RC (left) and NC (right).

Figure 3 shows the survivor curves estimated by the Kaplan-Meier estimator [5] and by the Markov model for both genders in RC and NC. The difference in LOS pattern between male and female residents following admission is apparent in both plots. However, in the case of NC, that difference diminishes as time passes, i.e., the LOS pattern in NC tends to be the same for both genders once residents settle in their new care environment.

\section{Conclusion}

In this paper, we have developed a model for the movement of elderly people in ILTC that takes into account residents' attributes. Using data provided by an English local authority, we have demonstrated in two case studies that such a model is able to give finer insights into the behaviour of the flow of residents in the care system, and can be a useful decision support tool for improving resident care management. In particular, our model provides quantitative support to the observations that male residents admitted to NC take more time to settle down $[1,2]$ and have poorer short-term survival prospect than female residents [2].

\section{Acknowledgement}

We thank Ms Teresa Temple, Mr Peter Crowther and the late Mr Terry Bucher from the Housing and Social Services Department of the London Borough of Merton for providing the data.

\section{References}

[1] A. Bebbington, R. Darton, and A. Netten, Care Homes for Older People (Volume 2): Admissions, Needs and Outcomes, Personal Social Services Research Unit (PSSRU), 
University of Kent, 2001.

[2] J. Cohen-Mansfield, M.S. Marx, S. Lipson, and P. Werner, "Predictors of mortality in nursing home residents", Journal of Clinical Epidemiology, 1999, 52:273-280.

[3] M.J. Faddy, and S.I. McClean, "Analysing data on lengths of stay of hospital patients using phase-type distributions", Applied Stochastic Models in Business and Industry, 1999, 15:311317.

[4] D.R. Fredkin, and J.A. Rice, "On aggregated Markov processes", Journal of Applied Probability, 1986, 23:208-214.

[5] E.L. Kaplan, and P. Meier, "Nonparametric estimation from incomplete observa-tions", Journal of the American Statistical Association, 1958, 53:465-481.

[6] I.C. Rothera, R. Jones, R. Harwood, A.J. Avery, and J. Waite, "Survival in a cohort of social services placements in nursing and residential homes: factors associated with life expectancy and mortality", Public Health, 2002, 116:160-165.

[7] D.R. Rowland, and A.M. Pollock, "Choice and responsiveness for older people in the 'patient centred' NHS”, British Medical Journal, 2004, 328:4-5.

[8] H. Xie, Modelling issues in long-term care: placement, survival and cost, $\mathrm{PhD}$ Thesis, University of Westminster, London, 2004.

[9] H. Xie, T.J. Chaussalet, and P.H. Millard, "A continuous-time Markov model for the length of stay of elderly people in institutional long-term care", Journal of the Royal Statistical Society A, 2005, 168:51-61. 